\title{
ADAPTAÇÃO NO MÉTODO DO PESO DA GOTA PARA DETERMINAÇÃO DA TENSÃO SUPERFICIAL: UM MÉTODO SIMPLIFICADO PARA A QUANTIFICAÇÃO DA CMC DE SURFACTANTES NO ENSINO DA QUÍMICA
}

\author{
João Lino Behring \\ Cremer S.A., CP 1508, 89010-971 Blumenau - SC \\ Mônica Lucas \\ Escola de Educação Básica Adolpho Konder, Rua Uberaba, 99, 89036-470 Blumenau - SC \\ Clodoaldo Machado e Ivonete Oliveira Barcellos* \\ Departamento de Química, Universidade Regional de Blumenau, CP 1500, 89010-971 Blumenau - SC
}

Recebido em 25/6/03; aceito em 26/11/03

\begin{abstract}
ADAPTATION OF THE DROP-WEIGHT METHOD FOR THE QUANTIFICATION OF SURFACE TENSION: A SIMPLIFIED APPARATUS FOR THE CMC DETERMINATION IN THE CHEMISTRY CLASSROOM. The discussion based on surfactant cleaning action is commonly used in chemistry classrooms for the comprehension of theoretical concepts related to surface tension and micelle formation. The experimental quantification of surface tension of aqueous solutions of surfactants provides the practical instruments for this discussion. The present paper describes a simplification on the common apparatus employed for the dropweight method, making the determination of surface tension accessible to any chemistry lab. The surface tension of various liquids and the critical micelle concentration, CMC, of three commercial surfactants were measured with this modified method, and proved to be consistent with literature values.
\end{abstract}

Keywords: surface tension; drop-weight method; theory/practice.

\section{INTRODUÇÃO}

A tensão superficial surge nos líquidos como resultado do desequilíbrio entre as forças agindo sobre as moléculas da superfície em relação àquelas que se encontram no interior da solução. As moléculas de qualquer líquido localizadas na interfase líquido-ar realizam um número menor de interações intermoleculares comparadas com as moléculas que se encontram no interior do líquido. A força resultante que atrai as moléculas da superfície de um líquido para o seu interior torna-se o principal obstáculo para a formação de bolhas, gotas e a nucleação de cristais em líquidos. Como estas forças de coesão tendem a diminuir a área superficial ocupada pelo líquido, observamos freqüentemente gotas adotarem a forma esférica. Pela mesma razão ocorre a formação dos meniscos, e a conseqüente diferença de pressões através de superfícies curvas ocasiona o efeito denominado capilaridade. A esta força que atua na superfície dos líquidos dá-se o nome de tensão superficial e, geralmente, quantifica-se a mesma determinando-se o trabalho necessário para aumentar a área superficial ${ }^{1}$.

Em geral, as discussões em sala de aula sobre a importância da tensão superficial restringem-se a sua relação com o que se convencionou chamar de "molhabilidade". Assim, quanto menor a tensão superficial maior a facilidade para um líquido se espalhar. Entretanto, as implicações deste fenômeno são bem mais amplas e estão diretamente relacionadas a muitas situações industriais, como os processos de fermentação, formação de gelo durante o resfriamento de alimentos ${ }^{2}$ e estabilidade de emulsões e espuma, bem como às funções vitais, como a tensão superficial nos pulmões. Neste último caso, os pulmões necessitam extrair o $\mathrm{O}_{2}$ do ar e passá-lo à corrente sanguínea, e o fazem através da presença do surfactante pulmonar, fosfolipídios, que baixa sensivelmente a tensão superficial das paredes dos alvéolos, facilitando a difusão do oxigênio ${ }^{3}$. Da mesma for-

*e-mail: iob@furb.br ma, o processo de tingimento nas indústrias têxteis necessita da adição de uma substância que diminua a tensão superficial (um tensoativo) das soluções dos corantes, facilitando a interação destes com o tecido a ser tinto, e aumentando a umectação das fibras ${ }^{4,5}$.

De fato, a aplicação de tensoativos, ou surfactantes, na indústria têxtil fornece uma ótima relação teoria-prática para a discussão da ação destes compostos sobre a tensão superficial. Devido à propriedade de reduzir a tensão superficial dos líquidos, os tensoativos podem ser classificados em: umectantes, detergentes, emulsionantes e solventes. As moléculas dos produtos tensoativos são constituídas por um grupo lipofílico e um grupo hidrofílico ${ }^{6}$.

Os tensoativos quando em solução, devido à presença do grupo lipofílico, ocupam preferencialmente a superfície do líquido, diminuindo a força de coesão entre as moléculas do solvente e, conseqüentemente, diminuindo a tensão superficial. Entretanto, após saturar a superfície, a adição de novas moléculas de tensoativo tem pouco efeito sobre o valor da tensão superficial, principalmente após atingirem uma certa concentração crítica (CMC), onde se formam espontaneamente agregados moleculares de dimensões coloidais, chamados micelas. A CMC depende da estrutura do tensoativo (tamanho da cadeia do hidrocarboneto) e das condições experimentais (força iônica, contra-íons, temperatura etc), e as discussões sobre sua formação, funções e relações com aplicações industriais é de extrema importância.

Portanto, a condução de experimentos que possibilitem a quantificação da tensão superficial, bem como o acompanhamento do efeito de tensoativos sobre esta propriedade, é fundamental em cursos de química, engenharia química e farmácia.

São diversos os métodos que podem ser empregados para a determinação da tensão superficial e estes são classificados em estáticos, dinâmicos e de desprendimento (ou separação). Dentre os métodos estáticos destacam-se o da ascensão capilar ${ }^{1,7}$, o da placa de Wilhelmy ${ }^{1,8}$ e do anel de DuNoüy ${ }^{1,9}$. Enquanto os dois últimos necessitam de equipamentos específicos, nem sempre disponíveis em 
cursos de graduação, o primeiro apresenta, em geral, erros experimentais bastante elevados, acima de $20 \%$. Isto se deve ao fato deste método, descrito por alguns autores como o mais preciso ${ }^{1}$, poder ser utilizado somente quando o ângulo de contato, formado pelo menisco e a parede do tubo, for zero, como no caso dos capilares. Entretanto, este diâmetro deve ser uniforme ao longo do tubo, restrição dificilmente atendida pelos capilares disponíveis comercialmente e com preços acessíveis. O método dinâmico da oscilação ${ }^{10}$ permite a determinação da tensão superficial em intervalos de tempo bastante curtos, mas trata-se de um dos métodos mais complexos existentes. Por outro lado, a determinação da tensão superficial fundamentada no desprendimento, como o método do peso da gota (um dos mais antigos) ${ }^{11}$ ou volume da gota, é convenientemente mais simples ${ }^{1}$.

No método do peso da gota geralmente emprega-se uma vidraria especial, o estalagmômetro, conforme desenho apresentado na Figura 1a, de modo a poder regular através da quantidade de líquido o tempo de formação da gota. No exato momento de desprendimento, a força exercida pelo peso da gota (m.g) é equilibrada pela tensão superficial $(\gamma)$ multiplicada pela circunferência $(2 . \pi . r)$ da gota formada. Desta forma, a tensão superficial pode ser calculada pela medida da massa (m) de uma gota do líquido, ou mesmo, pelo volume da gota (V) e a densidade do líquido $(\rho)$, de acordo com a Equação 1.

$\gamma=\frac{m \cdot g}{2 \pi \cdot r \cdot f}=\frac{V \cdot \rho \cdot g}{2 \cdot \pi \cdot r \cdot f}$

Há neste caso a necessidade de corrigir o volume da gota pela introdução de um fator de correção $(f)$, devido ao fato desta não se separar na forma esférica ${ }^{12}$. Uma vez mais, o aparato recomendado para a medição não é comum a laboratórios de química, e a sugestão de empregar uma microbureta acoplada a um dispositivo controlador de vazão não simplifica a metodologia.

Neste trabalho, testou-se o emprego de uma bureta comum para a obtenção das gotas (Figura 1b), e a validação da metodologia deu-se através da comparação da tensão superficial de vários líquidos e a CMC de tensoativos comerciais com valores da literatura e/ou mensurados por outros métodos. O principal objetivo é fornecer um experimento laboratorial altamente factível e que forneça baixos erros experimentais na determinação da tensão superficial, auxiliando desta forma o processo de ensino-aprendizagem na relação teoria-prática ${ }^{13}$. a)

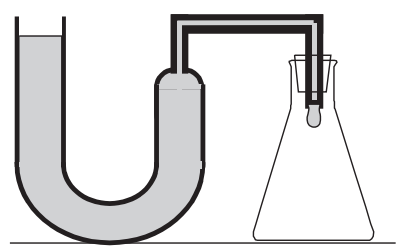

b)

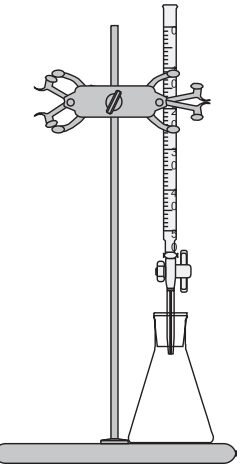

Figura 1. (a) Estalagmômetro e (b) bureta acoplada ao erlenmeyer, sistema empregado na determinação da tensão superficial do experimento

\section{PARTE EXPERIMENTAL}

\section{Equipamentos, materiais e reagentes}

Para a determinação da tensão superficial foram utilizadas buretas de 25 e $50 \mathrm{~mL}$ e erlenmeyers de $10 \mathrm{~mL}$. Os solventes empregados foram n-hexano, álcool etílico, acetato de etila, tetracloreto de carbono, todos de grau analítico e água destilada. Os surfactantes utilizados foram o brometo de n-hexadecil trimetil amônio, CTAB, $\left(\mathrm{CH}_{3}\left(\mathrm{CH}_{2}\right)_{15} \mathrm{~N}\left(\mathrm{CH}_{3}\right)_{3} \mathrm{Br}\right)$, catiônico; o polioxietileno(20)sorbitan monooleato, TWEEN 80, (R-( $\left.\left.\mathrm{C}_{17} \mathrm{H}_{33}\right) \mathrm{COO}\right)$, não-iônico e o lauril sulfato de sódio, SDS, $\left(\mathrm{C}_{12} \mathrm{H}_{25} \mathrm{NaO}_{4} \mathrm{~S}\right)$, aniônico.

Para fins de comparação, as tensões superficiais e a CMC dos surfactantes também foram quantificadas em um Tensiômetro Interfacial K8 Krüss, com anel de Pt-Ir de 20 mm de diâmetro.

\section{Metodologia}

A bureta foi fixada em suporte universal e acoplada a um erlenmeyer, de forma que a parte inferior à torneira ficasse toda dentro do recipiente coletor. Desta forma evita-se a influência de correntes de ar sobre a formação da gota. Com o auxílio da torneira é possível ajustar a queda das gotas em intervalos regulares de aproximadamente 1 min. É importante montar o aparato em um local com o mínimo de vibrações possível. Para cada solução foram coletadas 10 gotas, sendo os experimentos realizados em triplicata e a uma temperatura de $23{ }^{\circ} \mathrm{C}$. As massas foram determinadas em balanças analíticas. O raio (r) da circunferência da gota, na Equação 1, será idêntico ao raio interno da vidraria utilizada, quando esta não tocar a superfície do líquido. Dados publicados em $1975^{14}$ revelam uma relação linear entre a massa de uma gota e o raio da extremidade do tubo onde esta se formou. Assim, construímos a Equação 2 ( $\mathrm{n}=23$ pontos, $\mathrm{r}^{2}=0,9992$ ), de onde determina-se o raio (r) necessário para o cálculo da tensão superficial exclusivamente a partir da massa (m) de uma gota.

$r=-0,02815+3,81292 . m$

Conforme já mencionado, somente uma fração da gota se desprende do tubo durante o experimento, não formando uma circunferência perfeita. À medida que o orifício do tubo torna-se menor, a fração que se desprende torna-se maior e, a extrapolação para um tubo de diâmetro zero indica que, nestas condições, a gota se desprenderia integralmente. Harkins e Brown ${ }^{15}$ propuseram que o fator de correção (f) na Equação 1 depende da razão r/ $/{ }^{1 / 3}$, onde r é o raio na extremidade do tubo e $\mathrm{V}$ o volume de uma gota. Este volume pode ser determinado a partir da massa de uma gota e sabendo-se a densidade $(\rho)$ do líquido $(\rho=m / V)$. Infelizmente, nenhuma equação matemática se adapta à relação estabelecida (Figura 2) por Harkins e Brown ${ }^{15,16}$, que determinaram o valor do fator de correção para tubos de diferentes diâmetros, e o caminho mais fácil para se determinar o valor de $f$ é através de uma interpolação gráfica com valores de r/ $\mathrm{V}^{1 / 3}$ estando preferencialmente entre 0,6 e 1,2, onde a inclinação é menos acentuada.

\section{RESULTADOS E DISCUSSÃO}

Inicialmente, determinou-se o raio da bureta, medindo-se a massa de uma gota de água destilada e aplicando-se este resultado na Equação 2. Na seqüência, fez-se a rinçagem da bureta com o solvente orgânico em estudo, coletando-se a seguir 10 gotas deste líquido. A partir da determinação da massa de uma gota e empregando-se os dados da Figura 2 para determinação do fator de correção, pode-se determinar a tensão superficial dos líquidos em questão. A Figura 3 apresenta os resultados obtidos e compara os mesmos com valores da literatura. Estes últimos foram apurados dos dados publicados por Jasper ${ }^{17}$ e, por regressão linear, determinou-se a tensão superficial a $23{ }^{\circ} \mathrm{C}$.

Os erros relativos obtidos, em todos os casos, não ultrapassaram $10 \%$, e são bastante inferiores àqueles comumente registrados quan- 


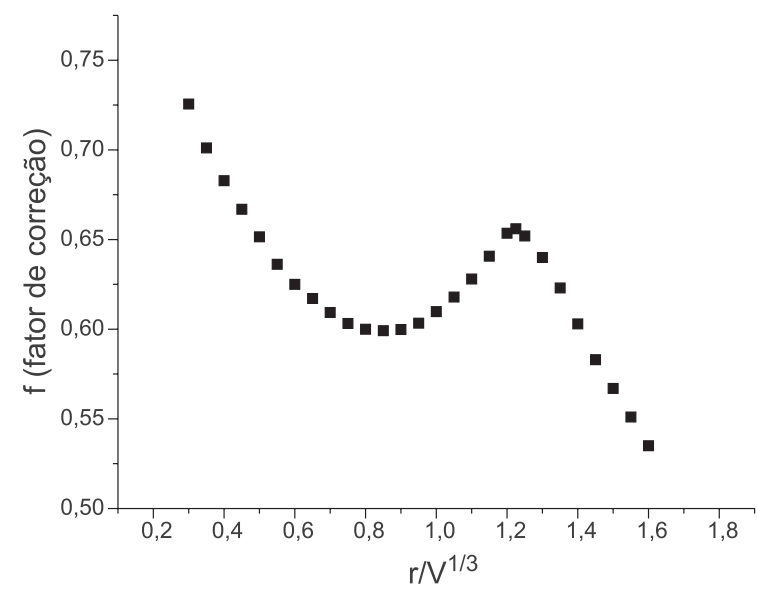

Figura 2. Dependência entre o fator de correção $(f)$ e a razão $r / V^{1 / 3}$

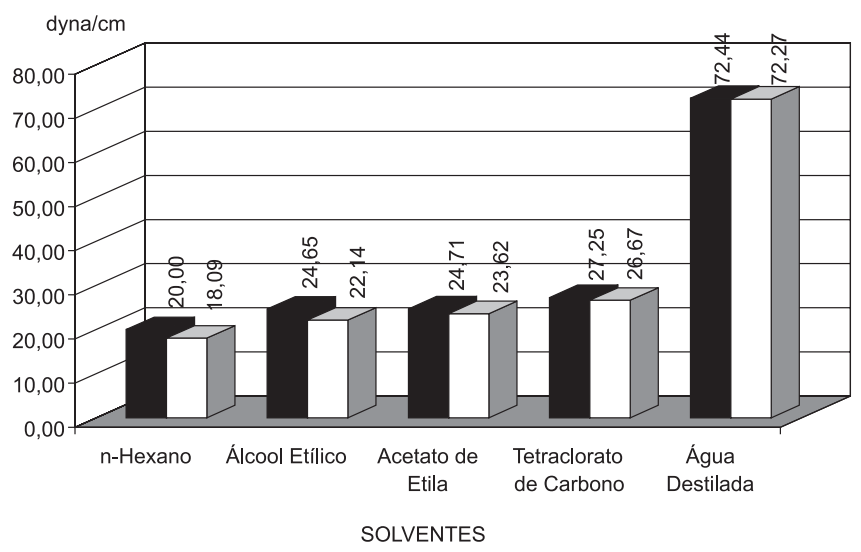

Figura 3. Valores de tensão superficial para diversos solventes determinados pelo: ( $\square$ ) método do peso da gota modificado em comparação com os ( respectivos valores da literatura, a $23{ }^{\circ} \mathrm{C}$

do se emprega o método da capilaridade para a determinação da tensão superficial. Da mesma forma, a bureta pode ser empregada na demonstração do efeito ocasionado pelos surfactantes sobre o valor da tensão superficial da água e, a partir do perfil da curva da tensão superficial versus a concentração de surfactante, determina-se a CMC do tensoativo. De fato, a mudança nas propriedades da solução, quando do início da formação das micelas, apresenta-se como um excelente exemplo para as discussões envolvendo fenômenos de superfície e colóides. Assim, a CMC pode ser determinada pelo estudo de várias propriedades, como condutividade elétrica, pressão osmótica, crioscopia, viscosidade, índice de refração, viragem de cor com corantes e espuma e, como empregado neste estudo, tensão superficial.

Quando presentes em soluções aquosas de baixas concentrações, as moléculas de surfactantes encontram-se na superfície da água, formando o filme superficial, ou no interior da solução, isoladas ou aos pares. Com o aumento da concentração formam-se as micelas, agregados de 50 ou mais monômeros, que adotam a forma esférica. Acima da CMC não existe um aumento significativo no número de partículas isoladas e, consequientemente, a força de coesão das moléculas da superfície é pouco modificada e não se observam mais efeitos significativos sobre a tensão superficial da solução.

A demonstração do efeito dos surfactantes sobre a tensão superficial pode ser realizada empregando-se tensoativos de uso comum, como o SDS, TWEEN ou CTAB (Figura 4).
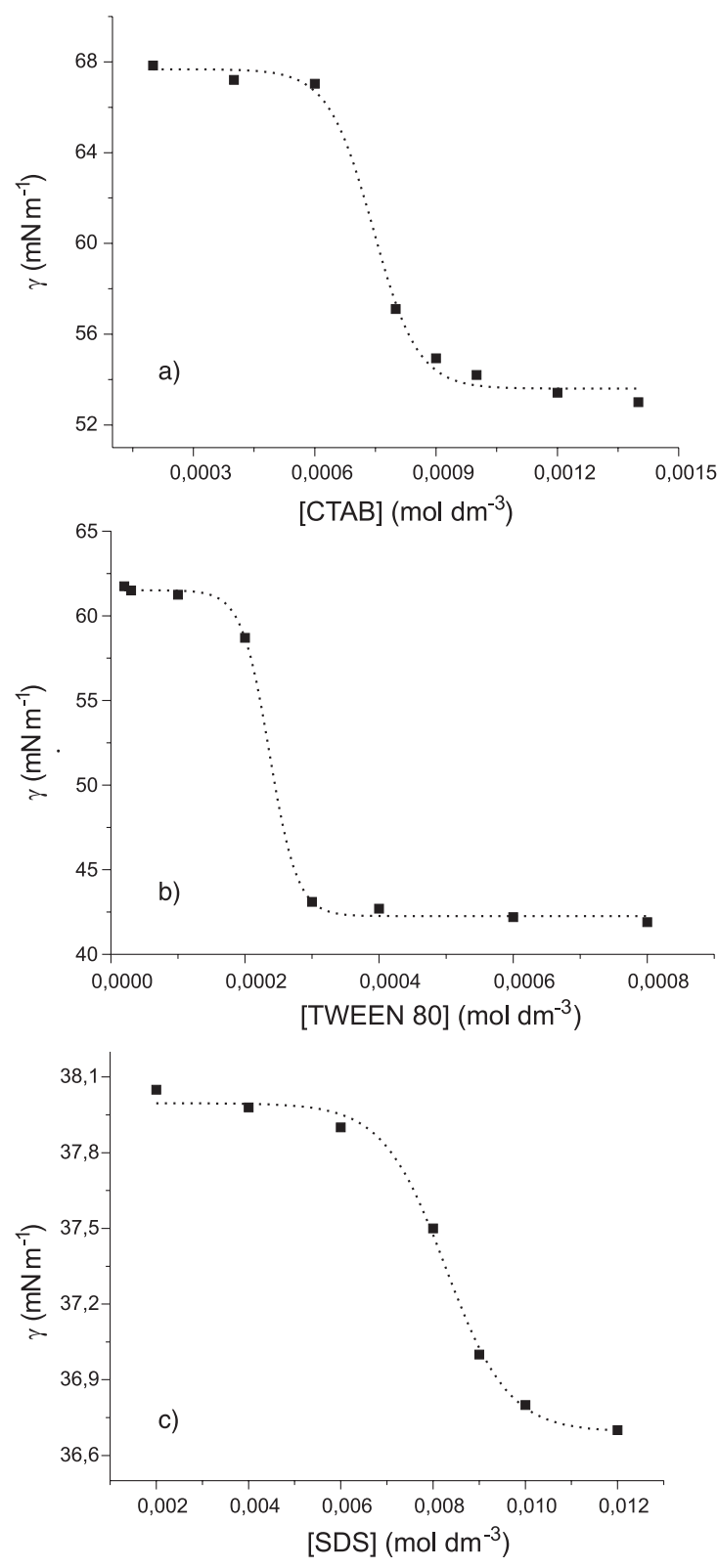

Figura 4. Efeito da concentração de surfactante sobre a tensão superficial para: (a) CTAB; (b) TWEEN 80 e (c) SDS, a $23{ }^{\circ} \mathrm{C}$

Em baixas concentrações, na parte esquerda dos gráficos, a concentração de surfactante não é suficiente para a saturação da superfície, e a tensão superficial praticamente não é alterada. Quando ocorre a formação de um filme superficial recobrindo toda a superfície do líquido, a tensão superficial da solução aquosa diminui acentuadamente, até o ponto onde se inicia a formação das micelas. A CMC é então determinada a partir do segundo ponto de inflexão, obtendose os seguintes valores experimentais: $8,2 \times 10^{-4} \mathrm{~mol} \mathrm{dm}^{-3}$ para o CTAB; $2,6 \times 10^{-4} \mathrm{~mol} \mathrm{dm}^{-3}$ para o TWEEN 80 e $9,1 \times 10^{-3} \mathrm{~mol} \mathrm{dm}^{-3}$ para o SDS. Os respectivos valores da literatura ${ }^{18}$ para estes surfactantes são $9,2 \times 10^{-4} ; 2,0 \times 10^{-4}$ e 7,5-8,5 x $10^{-3} \mathrm{~mol} \mathrm{dm}^{-3}$. A eficiência do método do peso da gota empregando-se exclusivamente uma bureta pode ser ainda comprovada pela comparação com os resultados obtidos quando um tensiômetro interfacial foi empregado para a quantificação da CMC, neste caso obtiveram-se os valores de: $10,0 \times 10^{-4} \mathrm{~mol} \mathrm{dm}^{-3}$ para o CTAB; $1,9 \times 10^{-4} \mathrm{~mol} \mathrm{dm}^{-3}$ para o TWEEN 80 e $10,0 \times 10^{-3} \mathrm{~mol} \mathrm{dm}^{-3}$ para o SDS, todos a $23^{\circ} \mathrm{C}$. 


\section{CONCLUSÃO}

A química é sabidamente uma ciência experimental e a realização de experimentos como instrumento para a discussão de conceitos teóricos é amplamente difundida. Contudo, restrições financeiras tendem a impor limites na efetivação de diversas práticas, principalmente com a contínua modernização dos instrumentos analíticos. O método do peso da gota já se apresenta como uma técnica extremamente simples para a determinação da tensão superficial e a modificação sugerida neste trabalho, torna-o acessível a laboratórios com condições mínimas de operação.

Alguns fatores precisam ser observados cuidadosamente durante a execução do experimento, pois de outra forma acarretarão em valores de tensão superficial bastante distintos daqueles presentes na literatura. Assim, é sabido que a temperatura influencia a tensão superficial, e recomenda-se efetuar o experimento em locais com temperaturas entre 15 e $25^{\circ} \mathrm{C}$. Desta forma os erros serão minimizados, pois tanto os dados empregados para a construção da Equação 2, quanto os apresentados na Figura 2 foram estabelecidos a $20^{\circ} \mathrm{C}$. A bureta deve ser fixada em local com o mínimo de vibrações, pois este fator pode acarretar no desprendimento prematuro da gota e, conseqüentemente, esta terá massa inferior àquela considerada ideal. Da mesma forma, a vidraria empregada deve possuir ponta plana, o que pode ser obtido lixando-se cuidadosamente a ponta da bureta. Outro fator fundamental para o sucesso do experimento é a formação lenta e controlada das gotas, a fim de garantir que a relação entre a massa da gota e o raio interno da bureta esteja de acordo com o proposto pela Equação 2. Por fim, cabe destacar que não há necessidade de se empregarem balanças analíticas para a determinação da massa da gota. Uma vez que muitos laboratórios não dispõem de tal equipamento, é possível utilizar balanças semi-analíticas, observando-se que um número maior de gotas deve ser coletado, a fim de minimizar o erro experimental advindo deste instrumento.

Os resultados aqui apresentados demonstram que técnicas fundamentadas em conceitos extremamente simples, onde a visualização dos aspectos teóricos se dá de forma direta, e as formulações matemáticas adquiriram sentido físico, podem fornecer resultados bastante precisos, motivando os alunos para estudos mais avançados. Finalmente, cabe destacar que a realização deste experimento possibilita uma ampla discussão sobre as aplicações industriais dos surfactantes nos mais diversos setores produtivos.

\section{AGRADECIMENTOS}

Os autores agradecem aos alunos dos cursos de graduação em química, engenharia química e farmácia da FURB pelas sugestões e comentários. Ao Prof. N. A. Debacher (UFSC) e à aluna de pósgraduação T. G. Rauen (UFSC) pelo uso do tensiômetro.

\section{REFERÊNCIAS}

1. Adamson, A. W.; Gast, A. P.; Physical Chemistry of Surfaces, $6^{\text {th }}$ ed., WileyInterscience: New York, 1997, cap. 2.

2. Rosenthal, A. J.; J. Chem. Educ. 2001, 78, 332

3. Gugliotti, M.; Química Nova na Escola, 2002, nº 16, 3.

4. Bracko, S.; Span, J.; Dyes Pigm. 2000, 45, 97.

5. Simon, B.; Kert, M.; Dyes Pigm. 2002, 54, 221.

6. Steinhart, M. R.; Revista Química Têxtil 2000, 61, 76.

7. Meyer, E. F.; Wyshel, G. M.; J. Chem. Educ. 1986, 63, 996.

8. Wilhelmy, L.; Ann. Phys. 1863, 119, 177.

9. Du-Nouy, P. L.; J. Gen. Physiol. 1919, 1, 521.

10. Srisankar, E. V.; Shah, J. P.; Narayan, K. S.; J. Chem. Educ. 1987, 64, 378.

11. Tate, T.; Philos. Mag. 1864, 27, 176.

12. Boucher, E. A.; Evans, M. J. B.; Proc. Roy. Soc. 1975, A346, 349.

13. Behring, J. L.; Lucas, M.; Machado, C.; Barcellos, I. O.; Resumos da $26^{a}$ Reunião Anual da Sociedade Brasileira de Química, Poços de Caldas, Brasil, 2003.

14. Manual do Engenheiro: Enciclopédia das Ciências e Arte do Engenheiro Arquiteto, $1^{\text {a }}$ ed., 1975, vol. 6, p. 222

15. Harkins, W. D.; Brown, F. E.; J. Am. Chem. Soc. 1919, 41, 499.

16. Lando, J. L.; Oakley, H. T.; J. Colloid Interface Sci. 1967, 25, 526.

17. Jasper, J. J.; J. Phys. Chem. Ref. Data, 1972, 1, 841.

18. Tokuda, J.; Ohura, R.; Text. Res. J. 1999, 69, 456. 